\title{
sciforum
}

Conference Proceedings Paper

\section{Do Sunspots Determine the Integrity of Satellite Navigation Signal? Fuzzy Logic Approach}

\author{
Karolina Krzykowska-Piotrowska ${ }^{1^{*}}$ \\ 1 Warsaw University of Technology; krzykowskakarolina@gmail.com \\ * Correspondence: krzykowskakarolina@gmail.com; Tel.: +48-22-234-1431
}

\begin{abstract}
There is a noticeable number of studies carried out on both the operational parameters of satellite systems and the satellite signal itself. The aim of such tests is to achieve the best possible results, inter alia, in terms of signal integrity. It also seems of key importance that the atmophere conditions, in any area of satellite signal usage, should not be underestimated due to its extensive impact. It has been proven, in the world literature, that the ionospheric factor is seriously limiting the operational use of the satellite navigation signal. The aim of the article is therefore to check whether the ionospheric factor, determined by solar activity, significantly affects the integrity of the satellite signal. For this purpose, a fuzzy logic approach will be used. Literature sources define two approaches to fuzzy control - descriptive and prescriptive. The first is based on the expert knowledge of the operator who knows from experience how to control the process. This is a traditional approach, not based on an existing model. The second approach assumes the existence of a stochastic or deterministic model and determines how to optimally control it. In the article, the traditional approach is closer to research. The model of the process determining the output (satellite signal integrity) as a function of the input (solar activity) is unknown. This means that the process is a black box. However, there is an available knowledge on how to control the process well, i.e. what control to choose for the current output.
\end{abstract}

Keywords: satellite signal; navigation; solar activity; ionosphere

\section{Introduction}

Already in year 2000, there was an interest in satellite systems applicable to various fields of engineering, especially to navigation. It turned out, however, that the use of a satellite navigation system entails the need to correct positioning errors caused by atmosphere or by signal reflection [1]. The article from 2016 proposed a method of eliminating GNSS multipath errors using an integrated measurement system and artificial neural networks [2]. Another source proposes a new method of improving positioning performance measured by pseudoranges in a degraded signal environment [3]. Importantly, the published materials also include researches related to the influence of external factors on the quality of the satellite signal. The team of C. Ordóñez Galán uses artificial neural networks to analyze the accuracy of measurements made with the GNSS technology when the GPS receiver is located in a forested environment [4].

The important issue of signal integrity has also been raised many times in literature. It is a measure of the probability that can be assigned to the correctness of the information provided by the navigation system. In many navigation satellite systems (eg. EGNOS European Geostationary Navigation Overlay Service), it is reflected in the Protection levels (PL) and Position errors (PE) values observed during system operation [5]. System integrity monitoring is possible thanks to RAIM (Receiver Autonomous Integrity Monitoring), which is a software that checks the correctness of 
information received from satellites using only signals from a given satellite navigation system, e.g. by comparing the position determined through different combinations of signals from different sets of observed satellites. According to literature sources [6-7], SAIM (Satellite Autonomous Integrity Monitoring) is to be an alternative to RAIM in the future.

In this article Author attempts to answer the question: Do sunspots determine the integrity of satellite navigation signal? Precisely, whether and to what extent selected external factors determine the possibility of using satellite navigation. For this purpose, the integrity measurements of the EGNOS signal (for the PRN120 and PRN126 satellites) performed in Warsaw in the first half of 2014 were used. Their dependence on solar activity during the same period was verified. Fuzzy sets were used to present the research results. The article has the following structure: introduction and state of the art (1), presentation of the research problem (2), research method (3), results and discussion (4), conclusions (5), literature.

\section{Research problem}

The operational limitation of satellite systems usage can be caused by various errors that are inherent in the operation of these systems. In general, errors in satellite systems can be divided into four groups:

- signal propagation errors, including:

o ionospheric errors;

o tropospheric errors;

o multi-path errors;

- relativistic errors;

- system operation errors, including: o satellite ephemeris errors;

o satellite clock errors;

- receiver errors, including DOP (Dilusion of Precision) error.

The passage of radio waves through the material medium can change their speed and frequency. On the way from the satellite to the receiver antenna, signal passes through the atmosphere, the properties of which change dynamically in a stochastic manner. The ionosphere, due to many researches, has the greatest influence on signal propagation.

Undoubtedly, Marconi, who in 1901 was the first to transmit radio signals across the Atlantic, contributed to discovery of the ionosphere. The location of receiving station out of the transmitting station's line of sight has given Marconi reason to believe that the electromagnetic wave is reflected from the atmosphere. The next step in the discoveries was to show that there is an inert electrical layer in the atmosphere. Solar radiation ionizes the gases present in this layer, resulting in clouds of free electrons. These electrons were influencing signals transmitted in the satellite system. Disruptions in the ionosphere cause the absorption and polarization of electromagnetic waves, which leads to interference with radio communication. The state of the ionosphere in context of satellite systems is determined by TEC (Total Electron Content) coefficient: the total number of electrons concentrated between two points along a column with a cross-section equal to $1 \mathrm{~m}^{2}$ and the base of rectangle. It is also called ionospheric delay or ionospheric refraction. The delay results from a change in the signal path, in addition to a change in the group and phase velocity of the wave (signal), and the frequency of the carrier wave. This leads to a change in time of signal displacement [8].

The state of the ionosphere is also influenced by solar activity, which is manifested by changes on the surface of the sun and its atmosphere. These changes cause fluctuations in the radiation that reaches the Earth in the form of electromagnetic waves, including light, and flux of particles emitted by the sun (solar wind). Solar activity also includes changes in the number and distribution of sunspots. The basic cycle of changes in solar activity is about 11 years, and in one cycle the sun changes the level of activity from a minimum to the next minimum [9].

The study of TEC variable, its modeling and prediction has become a popular problem in modern science in context of progress in satellite technology. Due to the proven significant influence 
of TEC on the quality of received satellite signal, much attention has been focused on TEC prediction, e.g. by using artificial neural networks [10-12].

Integrity - according to International Civil Aviation Organization (ICAO), is defined as a measure of confidence in the correctness of information provided by the system; includes the ability of system to deliver appropriate warnings (alerts) to the user at a predetermined time, which contain information on when the system should not be used. In context of integrity modeling, in order to determine if the position error is acceptable, an alert limit is set so that it is possible to reflect the maximum allowable position error that will not jeopardize the ongoing operation using a satellite signal (e.g. aircraft take-off). It should be remembered that navigation through satellite systems, and thus satellite signal, is provided simultaneously to many objects over a large area - often one or several continents. The impact of the satellite system integrity loss could be much greater than with conventional navigation methods. Sufficiently, early provided information about the loss of signal integrity should result in the resignation from the use of satellite navigation or the cessation of operations (e.g. take off or landing of an aircraft). Moreover, it is an individual and unique feature of satellite navigation to adapt the navigation capability to the changing constellation of satellites. The impact of changes in the space segment may be increased by an additional fault in the ground segment, for example damage of one of the components [13].

\section{Research methodology}

The analysis of phenomena related to the satellite signal is not possible without model analysis. Experimenting on the actual operation can lead to dramatic consequences. Therefore, numerous attempts are made to study the signal and the processes taking place in satellite systems during operation, which have a potential impact on its disturbance.

Fuzzy sets are a suitable tool for assessing the influence of solar activity on the integrity of a satellite signal. A fuzzy set is a mathematical object with a defined membership function that takes values from the range [0, 1]. The fuzzy set theory was introduced by Lotfi A. Zadeh in 1965 as an extension of the classical set theory. This reasoning is used when information is inaccurate or incomplete, which necessitates a decision under uncertainty. Knowledge about the phenomenon is subjective and is expressed through the opinion or descriptive research of an expert. It is informally referred to as linguistic uncertainty [14]. There are numerous references to the use of fuzzy sets in the literature, e.g. in context of air transport [15-17]. Their big advantage is the possibility of graphical presentation of research results, modeling uncertainty and examining the influence of many factors on the phenomenon.

The first stage of research procedure in this article included definition of fuzzy set and determination of linguistic variables membership function shape (table 1).

Table 1. Definition of fuzzy model including membership function

\begin{tabular}{cccccc}
\hline $\begin{array}{c}\text { Inputs/ } \\
\text { Outputs }\end{array}$ & $\begin{array}{c}\text { Linguistic } \\
\text { variable }\end{array}$ & Measure & $\begin{array}{c}\text { The range of } \\
\text { measurement values } \\
\text { from a given period }\end{array}$ & $\begin{array}{c}\text { Shape of the } \\
\text { membership } \\
\text { function }\end{array}$ & $\begin{array}{c}\text { Linguistic } \\
\text { values }\end{array}$ \\
\hline $\boldsymbol{x}_{\mathbf{1}}$ & Sunspots & $\begin{array}{c}\text { Number of } \\
\text { sunspots }\end{array}$ & $40-220$ & gauss curve & small \\
\hline $\boldsymbol{x}_{\mathbf{2}}$ & $\begin{array}{c}\text { Standard } \\
\text { deviation }\end{array}$ & $\begin{array}{c}\text { Value of } \\
\text { standard } \\
\text { deviation }\end{array}$ & $3,0-16,5$ & gauss curve & large \\
\hline $\boldsymbol{x}_{\mathbf{3}}$ & $\begin{array}{c}\text { Number of } \\
\text { observations }\end{array}$ & $\begin{array}{c}\text { Number of } \\
\text { observations }\end{array}$ & $6-21$ & gauss curve & medium \\
\hline $\boldsymbol{y}_{\mathbf{1}}$ & $\begin{array}{c}\text { Integrity of } \\
\text { PRN120 }\end{array}$ & PL - PE & & & large \\
\hline
\end{tabular}


The 3rd International Electronic Conference on Atmospheric Sciences (ECAS 2020), 16-30 November 2020; Sciforum Electronic Conference Series, Vol. 3, 2020

\begin{tabular}{|c|c|c|c|c|c|}
\hline & & & & & high \\
\hline \multirow[t]{3}{*}{$y_{2}$} & \multirow{3}{*}{$\begin{array}{c}\text { Integrity of } \\
\text { PRN126 }\end{array}$} & \multirow[t]{3}{*}{$P L-P E^{1}$} & \multirow[t]{3}{*}{$9,55-15,875$} & \multirow[t]{3}{*}{ gauss curve } & low \\
\hline & & & & & moderate \\
\hline & & & & & high \\
\hline
\end{tabular}

${ }^{1}$ PL - Protection Level, PE - Position Error. The higher difference between PL \& PE, the higher integrity of satellite signal. This measure is dedicated to EGNOS, due to [13].

An example of membership function shape for an input variable $\boldsymbol{x}_{\mathbf{1}}$ and output variable $\boldsymbol{y}_{\mathbf{1}}$ is shown through MATLAB Fuzzy Logic Toolbox in the Fig. 1.

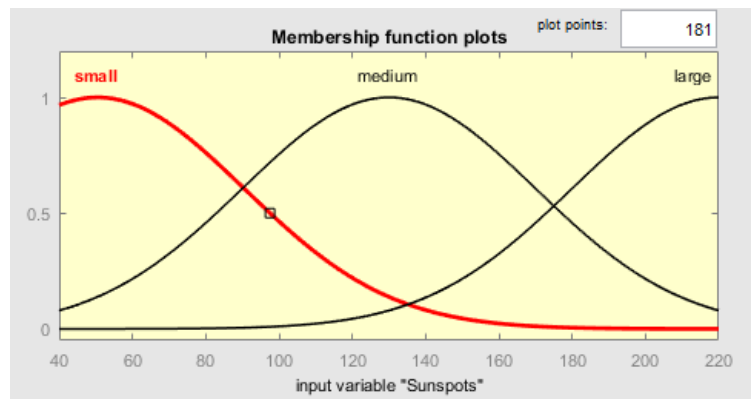

(a)

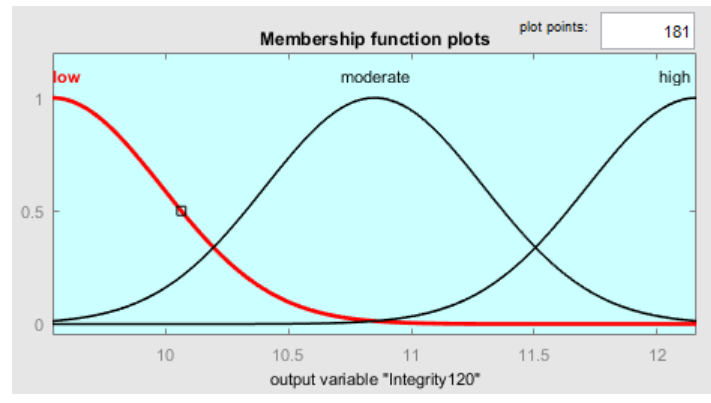

(b)

Figure 1. Example of the shape of the membership function for input variable $\boldsymbol{x}_{\mathbf{1}}$ (a) and output variable $\boldsymbol{y}_{\mathbf{1}}(\mathbf{b})$.

After this process, it is possible to finally formulate rules according to one of the architectures Mamdani or Takagi - Sugeno. The architecture of Mamdani was chosen in this research. There are different sources of rule making. Among them, the following can be distinguished: knowledge obtained from experts, scientific experience, literature sources, other research tools. The rule base according to the selected architecture is used for inference. The inference consists of determining the fuzzy value of the output variable, knowing the set of rules representing the relationships between the input and output variables, and some fuzzy values of the input variables. Having obtained a fuzzy value of the output variable, it should be defuzzified, that is, there should be found the corresponding strict (non-fuzzy) value. As the period of the first half of 2014 was used for the research, 181 measurements were available. Each of them constituted one rule in inference.

\section{Results \& discussion}

As a result of the conducted research, it can be clearly stated that the observations of solar activity are extremely important in context of integrity of satellite signal. In case of both, the PRN120 and PRN126 satellites, a large number of observations had a positive effect on the signal integrity (Fig. 2).

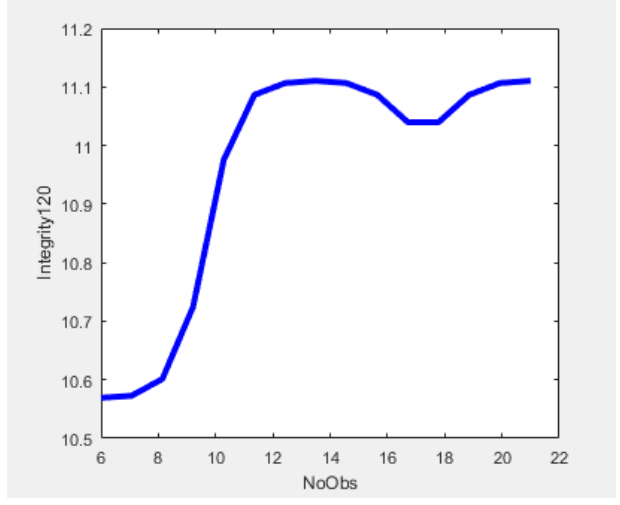

(a)

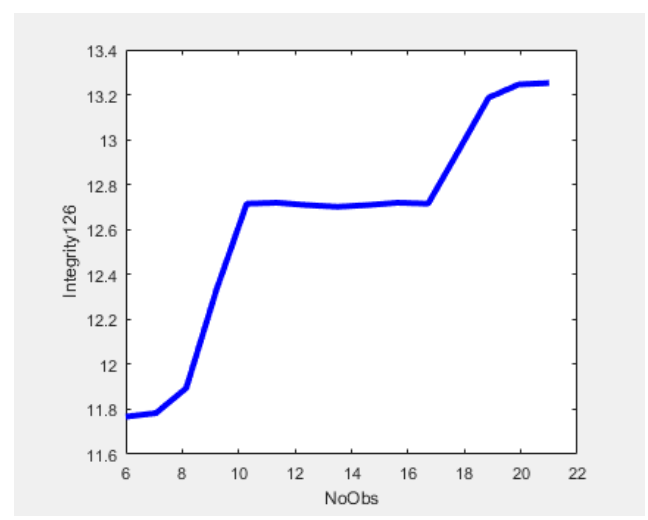

(b) 
The 3rd International Electronic Conference on Atmospheric Sciences (ECAS 2020), 16-30 November 2020; Sciforum Electronic Conference Series, Vol. 3, 2020

Figure 2. The influence of number of observations $\left(\boldsymbol{x}_{\mathbf{3}}\right)$ on integrity of PRN120 (a) and PRN126 (b).

Interestingly, the signal in the analyzed period obtained the highest integrity when the solar activity, measured by the number of sunspots, reached an average value in the range (101-160). This is reflected in the fact that there were definitely the most of such days. Fluctuations related to the number of sunspots negatively affected the signal quality for both large and small number of sunspots (Fig. 3).

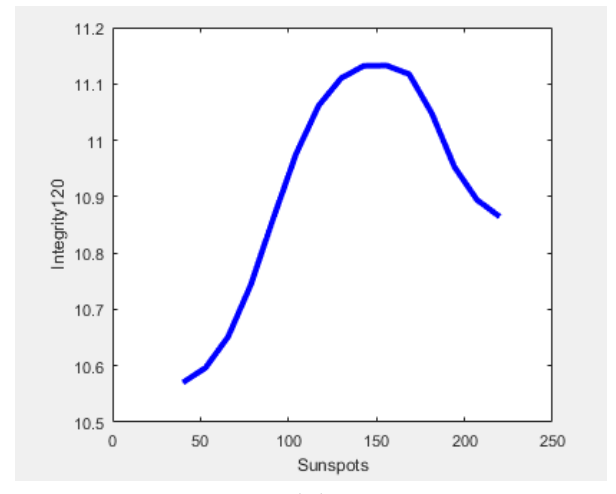

(a)

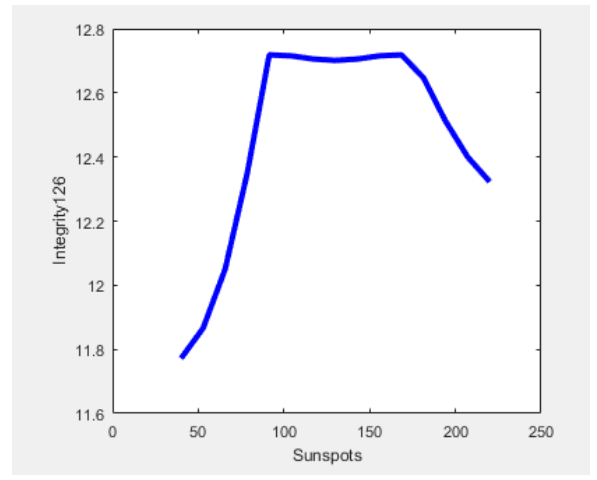

(b)

Figure 3. The influence of sunspots $\left(\boldsymbol{x}_{\mathbf{1}}\right)$ on integrity of PRN120 (a) and PRN126 (b).

In general, it can be concluded that the signal does not like changes and adapts slowly to them, and thus slowly acquires high integrity. This is also confirmed by the fact that integrity definitely decreased with increase of the standard deviation in both cases (PRN120, PRN126).

The last stage of the research is the process of defuzzification, i.e. searching for the non-fuzzy values of the variables that give the best result (highest integrity) - Fig. 4.

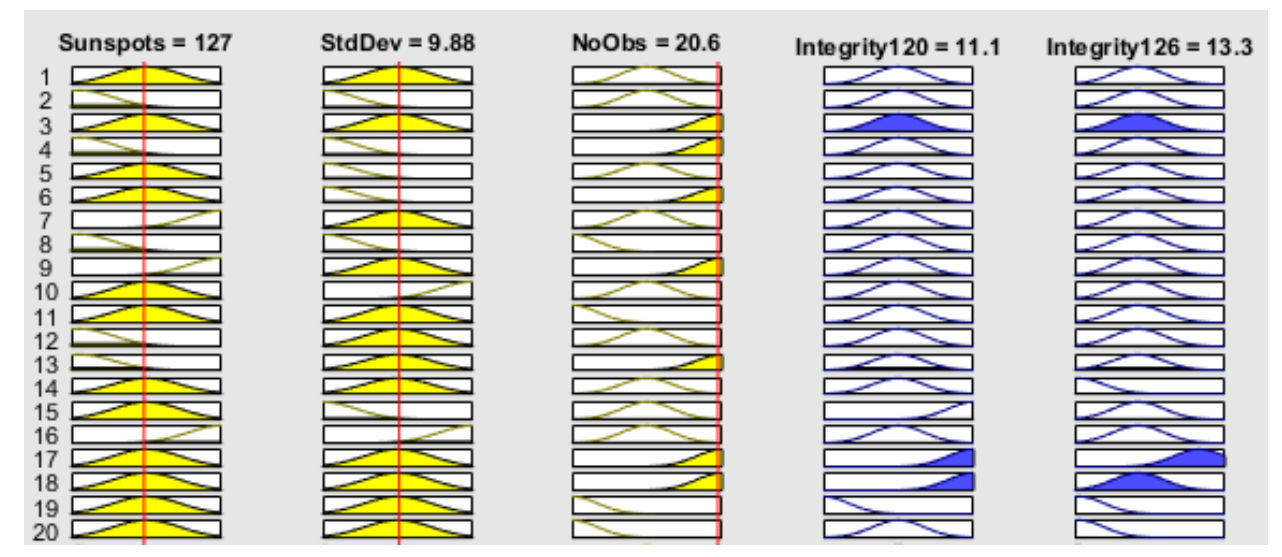

Figure 4. Graphical interpretation of the model defuzzification.

The combination from Fig. 4 was used as an example for the first 20 rules. A relatively high integrity value was achieved under certain input conditions: $\boldsymbol{x}_{1}=127, \boldsymbol{x}_{2}=9.88, \boldsymbol{x}_{3}=20.6$.

\section{Conclusions}

The issue of development and implementation of satellite systems has gained the attention of numerous scientific communities and providers of navigation services in recent years. This technology shows the innovative potential that meets the increasing needs of transport users. According to [18], by 2025, service providers will have the largest share of revenues from the sale of GNSS applications in General Aviation and business air transport. These assumptions meet the concept of SESAR (Single European Sky ATM Research) program to introduce the so-called flexible 
The 3rd International Electronic Conference on Atmospheric Sciences (ECAS 2020), 16-30 November 2020; Sciforum Electronic Conference Series, Vol. 3, 2020

airspace, eliminating restrictive flight plans and introducing $4 \mathrm{D}$ trajectories in air traffic. In view of the indicated plans and assumptions - it is justified to conduct research on predicting value of the signal integrity, and thus facilitate the decision to use it through navigation [19]. At the same time, it is worth emphasizing that the European GNSS Agency (GSA) constantly observes the EGNOS signal and although its performance in terms of parameters is getting better and better, the ionospheric factor was indicated as one of the main causes of interference [20].

Funding: This research received no external funding.

Conflicts of Interest: The authors declare no conflict of interest.

\section{References}

1. Ogaja, C.; Rizos, C.; Han, S. Is GPS good enough for monitoring the dynamics of high-rise buildings? 2nd Trans Tasman Surveyors Congress, Queenstown, New Zealand, 20-26 August, 2000.

2. Chi, L.; Zhuangsheng, Z. Study on real-time identification of GNSS multipath errors and its application. Aerospace Science and Technology 2016, (52), pp. 215-223. https://doi.org/10.1016/j.ast.2016.02.032

3. Hui, L.; Xu, L.; Shu, B.; Zhang, M.; Quia, C. A new method to improve the performance of multi-GNSS pseudorange positioning in signal-degraded environment. Advances in Space Research 2016, 58(4), pp. 577586. https://doi.org/10.1016/j.asr.2016.05.016

4. Rodriguez Perez, J. R.; Garcia Cortes, S.; Bernardo Sanchez, A.; Ordonez Galan, C. Analysis of the influence of forestry environments on the accuracy of GPS measurements by means of recurrent neural networks. Methematical and Computer Modelling 2013, 57(7-8), pp. 2016-2023. https://doi.org/10.1016/j.mcm.2012.03.006

5. Oliveira, J. M.; Tiberius, C. J. Quality Control in SBAS: Protection Levels and Reliability Levels. Journal of Navigation 2009, (63(3)). https://doi.org/10.1017/S0373463309005311

6. Xu, H.; Wang, J.; Zhan, X. GNSS Satellite Autonomous Integrity Monitring (SAIM) using inter-satellite measurements. Advances in Space Research 2011, (47(2011)), pp. 1116-1126. https://doi.org/10.1016/j.asr.2010.11.026

7. $\mathrm{Xu}, \mathrm{J}$; Y Yang, Y. GNSS receiver autonomous integrity monitoring (RAIM) algorithm based on robust estimation. Geodesy and Geodynamics 2016, 7(2), pp. 117-123. https://doi.org/10.1016/j.geog.2016.04.004

8. Januszewski, J. How the ionosphere affects positioning solution using terrestrial and satellite navigation systems. Communication in Computer and Information Science. Telematics in the Transport Environments, 2012, No. 329, pp. 249-257.

9. Lowell, W. E.; Davis, G. E. Solar cycles and their relationship to human disease and adaptability. Medical Hypotheses 2006, (67 (3)). doi:10.1016/j.mehy.2006.03.011

10. Yin, P.; Mitchell, C. N. Demonstration of the use of the Doppler Orbitography and Radio positioning Integrated by Satellite (DORIS) measurements to validate GPS ionospheric imaging. Advances in Space Research 2011, (48 (2011)), pp. 500-506. https://doi.org/10.1016/j.asr.2011.04.010

11. Habarulema, J. B.; McKinnell, L.-A.; Opperman, B. D. Towards a GPS-based TEC prediction model for Southern Africa with Feed forward networks. Advances in Space Research, 2009, (44 (2009)), pp. 82-92. https://doi.org/10.1016/j.asr.2009.02.016

12. Paul, A.; Sur, D. Comparison of standard TEC models with a Neural Network based TEC model using multistation GPS TEC around the northern crest of Equatorial Ionization Anomaly in the Indian longitude sector during the low and moderate solar activity levels of the 24th(...). Advances in Space Research 2013, 52(5), pp. 810-820. https://doi.org/10.1016/j.asr.2013.05.020

13. International Civil Aviation Organization. Annex 10 to the Convention on International Civil Aviation. Volume I Radio Navigation Aids. 2006.

14. Zadeh, L. A. Fuzzy sets. Information and Control 1965, (8), pp. 338-353.

15. Grzesik, N. Fuzzy sets in aircraft system efficiency evaluation. Aircraft Engineering And Aerospace Technology 2015, 88(6). doi: 10.1108/AEAT-03-2015-0084

16. Wang, C. Y. Topological characterizations of generalized fuzzy rough sets. Fuzzy Sets and Systems 2017, (312), pp. 109-125. https://doi.org/10.1016/j.fss.2016.02.005

17. Omolbanin, Y.; Dick, S. A systematic review of complex fuzzy sets and logic. Fuzzy Sets and Systems 2017. https://doi.org/10.1016/j.fss.2017.01.010 
The 3rd International Electronic Conference on Atmospheric Sciences (ECAS 2020), 16-30 November 2020;

Sciforum Electronic Conference Series, Vol. 3, 2020

18. Aguilera, C.; Lorenzo, J. M. EGNOS in aviation: strategy and implementation status. EGNOS Service Provision Workshop; 2017; Athens.

19. Krzykowska, K.; Dudek, E. How to prevent loss of satellite navigation continuity caused by nimbostratus? Concept for Polish ATM, Transportation Research Procedia 2019, Volume 43, pp. 63-73. https://doi.org/10.1016/j.trpro.2019.12.020

20. European GNSS Agency (GSA). SBAS Independent Assessment. EGNOS Annual Workshops; 2017; Athens.

(C) 2020 by the authors. Submitted for possible open access publication under the terms and conditions of the Creative Commons Attribution (CC BY) license (http://creativecommons.org/licenses/by/4.0/). 